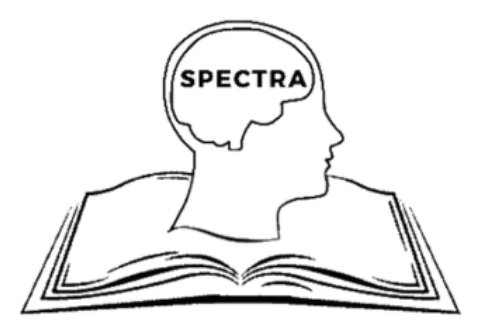

Spectra Undergraduate Research Journal

Volume 1, Issue 2

Office of Undergraduate Research

University of Nevada, Las Vegas

digitalscholarship.unlv.edu/spectra/

\begin{tabular}{|c|}
\hline $\begin{array}{c}\text { Category } \\
\end{array}$ \\
\hline Health \& Natural Sciences \& Engineering $>$ Natural Sciences $>$ Biology \\
\hline Received \\
\hline March 31, 2021 \\
\hline Accepted \\
\hline July 19, 2021 \\
\hline Published \\
\hline August 13, 2021 \\
\hline Article Title \\
\hline $\begin{array}{l}\text { A Timeline of Discovery and Current Research on Primary Open-Angle Glaucoma and Emergence } \\
\text { of Potentially Permanent Treatment Solutions }\end{array}$ \\
\hline Authors \\
\hline Yonosuke DeJesus $(\mathrm{YD})^{1 *}$ and Guadalupe Moreno Ceballos $(\mathrm{GMC})^{1,2}$ \\
\hline Author Affiliations \\
\hline $\begin{array}{l}{ }^{1} \text { School of Life Sciences, University of Nevada, Las Vegas, Las Vegas, NV, USA. } \\
{ }^{2} \text { Department of Psychology, University of Nevada, Las Vegas, Las Vegas, NV, USA. }\end{array}$ \\
\hline Corresponding Author \\
\hline *Yonosuke DeJesus, aokiy1@,unlv.nevada.edu \\
\hline Author Contributions \\
\hline $\begin{array}{l}\text { YD: Conducted a literature search and review, fully drafted the manuscript, made significant } \\
\text { revisions to the manuscript, generated figures, and approved the version to be published } \\
\text { GMC: Conducted a literature search and review, made critical revisions to the manuscript, } \\
\text { incorporated figures, implications of the results, and approved the version to be published }\end{array}$ \\
\hline Copyright \\
\hline $\begin{array}{l}\text { (c) Articles in Spectra are freely available under a Creative Commons Attribution } \\
\text { License (CC BY 4.0) which allows others to re-use the work without permission as } \\
\text { long as the work is properly cited. }\end{array}$ \\
\hline ISSN \\
\hline $2766-7227$ \\
\hline Data Availability Statement \\
\hline $\begin{array}{l}\text { The authors of this article confirm that all included literature review information is fully available } \\
\text { without restrictions. }\end{array}$ \\
\hline Conflicts of Interest \\
\hline The authors declare that no conflicts of interest exist. \\
\hline Ethical Considerations \\
\hline $\begin{array}{l}\text { Given this is a project-based literature review, and did not involve human or animal subjects, no IRB } \\
\text { or IACUC approval was needed. No data presented in this paper has been derived from participants } \\
\text { and all program elements are publicly shared. }\end{array}$ \\
\hline Funding \\
\hline No funding was used to conduct this research. \\
\hline Recommended Citation \\
\hline $\begin{array}{l}\text { DeJesus, Y. \& Moreno Ceballos, G. (2021). A timeline of discovery and current research on primary } \\
\text { open-angle glaucoma and emergence of potentially permanent treatment solutions. Spectra } \\
\text { Undergraduate Research Journal, 1(2), 24-35. https://doi.org/10.9741/2766-7227.1007 }\end{array}$ \\
\hline
\end{tabular}




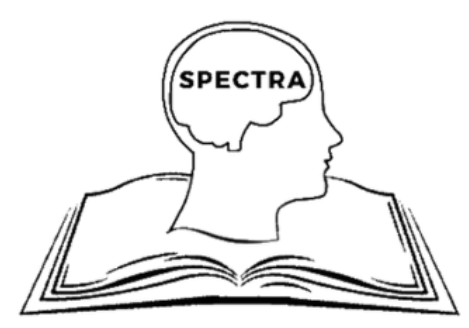

Spectra Undergraduate Research Journal

Volume 1, Issue 2

Office of Undergraduate Research

University of Nevada, Las Vegas

digitalscholarship.unlv.edu/spectra/

\title{
A Timeline of Discovery and Current Research on Primary Open-Angle Glaucoma and Emergence of Potentially Permanent Treatment Solutions
}

Yonosuke DeJesus ${ }^{1}$ and Guadalupe Moreno Ceballos ${ }^{1,2}$

\section{Author Affiliations:}

${ }^{1}$ School of Life Sciences, University of Nevada, Las Vegas, Las Vegas, NV, USA.

${ }^{2}$ Department of Psychology, University of Nevada, Las Vegas, Las Vegas, NV, USA.

\begin{abstract}
This research examines the timeline of the discovery and research of Primary Open-Angle Glaucoma (POAG). By reviewing the literature on genetic and molecular mechanisms, we aim to emphasize a long-term treatment solution (iSTENT) to mitigate intraocular pressure (IOP) related to POAG etiology and disease progression. POAG is a multifactorial, autosomal dominant, adult-onset eye disease wherein the optic nerve cells become damaged due to a buildup of excess aqueous humor, resulting in increased IOP. Consequently, POAG leads to progressive loss of peripheral vision and is the leading cause of blindness in the US. Due to the multifactorial nature of glaucoma's inheritance pattern and disease pathology, over 20 disease-associated loci have been implicated with POAG and its mechanisms remain relatively obscure. POAG remains a hotbed for multifactorial genetic research and the relationships between heterogeneity and environmental/genetic factors. To develop a more permanent solution for Glaucoma, we highlight a surgical insertion of a stent in the trabecular meshwork providing long-lasting dilation to the blocked drainage channels implicated with increased IOP and POAG progression. This procedure would allow for aqueous humor outflow from the eye and subsequent reduction of pressure-induced retinal ganglion cell damage. POAG's progressive loss of vision due to polygenic inheritance and environmental factors is currently experienced by over 2.25 million Americans and will continue to grow as POAG-associated genes are maintained in subsequent generations. Therefore, a complete understanding of the history, etiology, and genetic mechanisms is critical for the development of effective and long-lasting treatment options.
\end{abstract}

Keywords: genetics, multifactorial disease, MYOC (gene), intraocular pressure (IOP), blindness, iSent Medical Device

In a healthy eye, fluid called aqueous humor is secreted into the posterior chamber (behind the iris) via the ciliary processes attached to the ciliary body. The aqueous humor then crosses the pupil to provide nutrients to the cornea (clear portion) in the anterior chamber (in front of the iris) of the eye (Llobet et al., 2003). Lastly, the eye fluid exits the anterior chamber through the trabecular meshwork tissue (TM) and subsequently through Schlemm's canal (See Figure 1 for anatomy of the eye).

In contrast, Primary Open-Angle Glaucoma (POAG) is an adult-onset eye disease that causes progressive blindness, typically beginning with a gradual loss of peripheral vision. This disease is often a result of defects in the trabecular meshwork preventing aqueous humor from properly exiting the 
eye cavity through drainage channels. This inability of the aqueous humor to drain properly causes a build-up in intraocular pressure (IOP), referring to fluid pressure within the eye. As IOP increases due to blocked or collapsed drainage channels in the trabecular meshwork, retinal ganglion cells (optic nerve cells) become severely damaged or undergo apoptosis (cell death) resulting in the permanent progressive loss of vision (See Figure 2 for an illustration of the development of glaucoma). The "Open-Angle" portion of the POAG name refers to the presence of space between the iris and cornea.

From a genetic perspective, POAG is a multifactorial disease with complex inheritance meaning that multiple genes have been known to cause or affect the expression of the disease. Additionally, the penetrance and expressivity of POAG can vary drastically from patient to patient (Gemenetzi et al., 2012). In other words, people who carry the POAG mutations may or may not exhibit signs and symptoms of the disease. Genetic and environmental modifiers of POAG interact to contribute to the notable complexity of its disease etiology, severity, and inheritance patterns. Some of the most common non-genetic modifiers include race, untreated high blood pressure, cigarette smoking, family history of glaucoma, diabetes, and being nearsighted. Currently, there is no permanent cure for POAG and current treatment options require daily use of specialized IOP reducing eye drops or multiple follow-up procedures. Thus, using current and historical research, we investigate a molecular mechanism of POAG to emphasize a permanent treatment solution to IOP-induced retinal ganglion cell injury/death using an in-development medical device known as the iStent.

\section{Literature Review}

\section{Early History:}

\section{BCE Hippocrates}

Taking a look at the history, Glaucoma has been prevalent in the human population dating back to 400BCE where it was mentioned by Hippocrates, the Father of Modern Medicine. He referred to this condition as "Glaykoseis" - blindness that occurs in the elderly (Leffler, 2015).

502-575 CE Aetius of Amida
By 500CE a Byzantine physician, Amida, grouped it under the term "Amaurosis", a categorization used to describe eye diseases that caused severe vision impairment as a result of increased eye pressure on the optic nerve in patients with eyes that appeared normal (Leffler, 2015).

\section{1-1300 Benevenutus Grassus}

12th-century optometrist Benevenutus Grassus coined the term "gutta serena" to describe incurable blindness caused by an obstructed optic nerve (Leffler, 2015).

\section{Invention of Ophthalmoscope}

In the 1850s a breakthrough in diagnosis occurred with the invention of the ophthalmoscope. For the first time in history, this revolutionary device allowed physicians to visually identify a degraded optic nerve and finally led to the inception of the current name "Glaucoma" (Leffler, 2015).

1856 Albrecht von Graefe

Albrecht von Graefe introduced iridectomy as one of the earliest forms of glaucoma treatment. This operation involves surgical removal of a segment of the iris allowing for drainage of excess fluid from the eye. Graefe then went on to invent the tonometer, a tool currently used to measure the eye pressure of glaucoma patients. Graefe is also credited with the implementation of visual field testing, a commonly used practice used to identify and diagnose glaucoma (Leffler, 2015).

\section{Trabeculectomy Introduced}

In 1960 Trabeculectomy, a procedure that involves surgical removal of a small portion of the trabecular meshwork to drain aqueous humor was introduced. This procedure shifted several future procedures towards the trabecular meshwork and its drainage channels as a new, more efficient target for surgical intervention (Razeghinejad, 2011).

1967 Discovery of Multifactorial Disease Etiology

The study by Mansour, F. P., described POAG's locus heterogeneity by performing parentoffspring transmission comparisons (i.e. pedigree). This study further concluded polygenic inheritance in POAG, with variable age of onset and expressivity. This discovery confirmed that multiple genes along with environmental factors all played a collective role in disease manifestation and severity (Armaly, 2017). 1996 Prostaglandin Medications Introduced 
First eye drops that reduce intraocular pressure are introduced and are still currently the first line of medical therapy for glaucoma. Prostaglandins act by activating receptors that can trigger a restructuring of the extracellular matrix to allow for aqueous outflow (Fingeret, 2001).

1997 Discovery of First Disease-Associated Gene MYOC

A study by Stone et al performed SequenceTagged Sites (STS) on candidate genes based on affected familial association studies of POAG. Radiation hybrid gene mapping was used to suggest the presence of the MYOC disease-associated gene near the candidate gene markers (Stone et al., 1997).

1997 Laser Trabeculoplasty Introduced

Laser-based surgery, in which a laser is used to cut damaged regions of the trabecular meshwork, begins being practiced by optometrists. This procedure currently remains the most frequently used glaucoma treatment (Razeghinejad, 2011).

\section{Current Research:}

2009 Identification of 3 susceptible Loci in Japanese Populations

3 susceptible loci on chromosomes 1, 10, and 12 were identified using a genome-wide SNP association study of 418 POAG patients. Glaucoma was found to exhibit familial aggregation (autosomal dominant; $50 \%$ inheritance), with variable penetrance \& expressivity amongst individuals and various ethnic groups (African Americans, Japanese and Northern Europeans) (Nakano et al., 2009).

2009 Investigation of MYOC Hotspots on Glaucoma Etiology

This 2009 research found several POAGassociated mutations are often a result of Single Nucleotide Polymorphisms (SNPs). The MYOC gene which encodes the protein myocilin, produced in the trabecular meshwork tissue, was discovered to be a key hotspot in Glaucoma etiology.

The exact function of myocilin is unknown. However, various mutations of MYOC have been associated with retinal ganglion damage and death. This study indicated that the MYOC gene segment, in particular, accrues several point mutations. These point mutations occur most commonly as a result of spontaneous or mutagen-induced DNA damage, causing errors in DNA replication/repair by DNA polymerase (Kwon et al., 2009).

2013 Research Implicates Dual Leucine Zipper Kinase as a Key Mediator of Retinal Ganglion Cell Apoptosis

A 2013 Research identified several important myocilin domains and some of their normal roles. From the illustrations above, dual Leucine zipper kinase in the $\mathrm{N}$-terminus was discovered to be a highly important signaling component during axonal damage \& retinal ganglion cell death, while the C-terminal domains in myocilin functioned to direct proteins to peroxisomes for oxidation reactions (Welsbie et al., $\underline{2013}$ ).

2013 Research Implicates Dual Leucine Zipper Kinase as a Key Mediator of Retinal Ganglion Cell Apoptosis

A 2013 Research identified several important myocilin domains and some of their normal roles. From the illustrations above, dual Leucine zipper kinase in the $\mathrm{N}$-terminus was discovered to be a highly important signaling component during axonal damage \& retinal ganglion cell death, while the C-terminal domains in myocilin functioned to direct proteins to peroxisomes for oxidation reactions (Welsbie et al., $\underline{2013}$ ).

2015 Psychosocial Study on Glaucoma-Associated Visual Acuity \& Field Loss

This study found that glaucoma-related loss of visual acuity and field had significant psychosocial effects on the patients. This was determined using a quality of life questionnaire of 192 patients. Results indicated that glaucoma patients had $63 \%$ greater anxiety, $71 \%$ lower self-image, $38 \%$ less psychological well-being \& $32.4 \%$ reduced confidence in health care compared to negative controls (Chan et al., 2015).

2017 Genome-wide Association Identifies 16 total POAG-associated Gene Loci

Genome-wide association studies for different types of adult-onset glaucoma have identified 16 total POAG-associated loci involved in a variety of important biological functions including; cytokine signaling, lipid metabolism, membrane biology, and ocular development.

This discovery truly emphasizes the complex, multifactorial nature of this disease. Several of these seemingly unrelated genetic loci could be acting together or independently to reduce or exacerbate the condition of this glaucoma. This relative abundance of 
genetic modifiers coupled with environmental factors contributes to the notable heterogeneity of glaucoma manifestation (multifactorial) (Bailey et al., 2016).

Potential Long-Term Treatment (iStent): Due to the complex, multifactorial nature of glaucoma, genetic intervention and long-term IOP reducing therapy may present significantly challenging biological/financial barriers (Wiggs et al., 2017). Therefore, consideration of more permanent solutions such as the iStent or other stent-based procedures could provide efficient protection against progressive loss of vision and offer additional long-term health and financial benefits.

Surgical insertion of the iStent ${ }^{\circledR}$ (stent) into the trabecular meshwork allows aqueous humor to drain freely into the Schlemm's canal from the anterior chamber, reducing IOP-induced retinal ganglion cell damage and death (Resende et al., 2016). This procedure is known as minimally invasive glaucoma surgery (MIGS) has shown notably low complication rates and long-term reduction in unmedicated IOP.

A 13-month follow-up of 39 POAG patients treated with the insertion of 2 iStents has exhibited a mean unmedicated IOP reduction of $25.3 \pm 1.8 \mathrm{mmHg}$ preoperatively to $17.1 \pm 2.2 \mathrm{mmHg}(32.4 \%)$ (Resende et al., 2016). Due to the notable heterogeneity of glaucoma, it is an important model for the study of multifactorial disease etiology.

\section{Discussion}

POAG's incurable progressive loss of vision is currently experienced by over 2.25 million Americans and remains the leading cause of blindness in the US. With a long history of prevalence in individuals dating back to as early as 400 BCE (Leffler, 2015), this disease possesses multifactorial characteristics contributing to the complexity of this disease's penetrance, expressivity, and overall inheritance patterns.

POAG's influence from both genetic and environmental modifiers also diminishes the accurate evaluation of POAG prognosis. Patients typically exhibit varying severity of symptoms with individuals who may even be completely unaware of their condition due to its often gradual progression. Furthermore, the incurable progressive blindness due to POAG has also been shown to additionally cause a decline in the mental state of the patients, highlighting several of the long-term, non-physical implications of this disease (Zhou et al., 2016).

Current research has also identified several of the proteinaceous, structural defects of POAGimplicated gene products such as Myocilin from the MYOC gene. Discovery and analysis of such defective conformational structures of POAG-associated proteins may unlock breakthrough permanent treatment avenues (Welsbie et al., 2013). With the introduction of the iStent medical device, we believe this device along with other similar stent-based devices could provide long-term financial and clinical benefits. The iStent in particular is promising as it bypasses the genetic aspects of POAG and intervenes at the physical level (direct insertion into the eye) with little to no follow-ups (Resende et al., 2016).

A comprehensive understanding of POAG's unique genetic characteristics, history, molecular mechanisms, and current research may provide key insight to a cure for IOP-induced retinal ganglion cell damage. Furthermore, because POAG remains so prevalent in the United States, a complete understanding of its history and etiology is crucial for the development of long-term, financially viable, treatment avenues.

\section{Acknowledgments}

We want to thank our research advisor and faculty mentor Dr. Kathryn Rafferty for her continued guidance and support throughout our entire research process. We want to also thank the staff at the UNLV Office of Undergraduate Research (OUR) for providing a wealth of resources to enhance our research through the presentation at the OUR Undergraduate Research Symposium to the 2021 NCRC event.

\section{References}

Armaly M. F. (1967). Inheritance of dexamethasone hypertension and glaucoma. Archives of ophthalmology (Chicago, Ill. : 1960), 77(6), 747-751.

https://doi.org/10.1001/archopht.1967.009800 20749006

Bailey, J. N., Loomis, S. J., Kang, J. H., Allingham, R. R., Gharahkhani, P., Khor, C. C., Burdon, K. P., Aschard, H., Chasman, D. I., Igo, R. P., Jr, Hysi, P. G., Glastonbury, C. A., Ashley- 
Koch, A., Brilliant, M., Brown, A. A., Budenz, D. L., Buil, A., Cheng, C. Y., Choi, H., Christen, W. G., ... Wiggs, J. L. (2016). Genome-wide association analysis identifies TXNRD2, ATXN2 and FOXC1 as susceptibility loci for primary open-angle glaucoma. Nature genetics, 48(2), 189-194. https://doi.org/10.1038/ng.3482

Chan, E. W., Chiang, P., Liao, J., Rees, G., Wong, T.Y., Lam, J.S.H., Aung, T., \& Lamoureux, E. (2015). Glaucoma and Associated Visual Acuity and Field Loss Significantly Affect Glaucoma-Specific Psychosocial Functioning. Ophthalmology (Rochester, Minn.), 122(3), 494-501.

https://doi.org/10.1016/j.ophtha.2014.09.030

Fingeret, M. "Prostaglandins may benefit glaucoma patients as first-line medication." Primary Care OptometryNews: 2001. Retrieved from:

https://www.healio.com/news/optometry/2012 0225/Prostaglandins-may-benefit-glaucomapatients-as-first-line-medication

Gemenetzi, M, Yang, Y, \& Lotery, A.J. (2012). Current concepts on primary open-angle glaucoma genetics: A contribution to disease pathophysiology and future treatment. Eye (London), 26(3), 355-369. https://doi.org/10.1038/eye.2011.309

Kwon, Y. H., Fingert, J. H., Kuehn, M. H., \& Alward, W. L. M. (2009). Primary OpenAngle Glaucoma. The New England Journal of Medicine, 360(11), 1113-1124. https://doi.org/10.1056/NEJMra0804630

Leffler, C. T., Schwartz, S. G., Giliberti, F. M., Young, M. T., \& Bermudez, D. (2015). What was Glaucoma Called before the 20th Century? Ophthalmology and Eye Diseases, 7. https://doi.org/10.4137/OED.S32004

Lin, W., \& Kuang, H. (2014). Oxidative stress induces autophagy in response to multiple noxious stimuli in retinal ganglion cells. Autophagy, 10(10), 1692-1701. https://doi.org/10.4161/auto.36076

Llobet, A., Gasull, X., \& Gual A. (2003). Understanding trabecular meshwork physiology: A key to the control of intraocular pressure? NIPS, 18(5), 205-209.

https://doi.org/10.1152/nips.01443.2003

N. A. (2020, August 18). Early-onset glaucoma.

Medline Plus Genetics.

https://medlineplus.gov/genetics/condition/earl y-onset-glaucoma/

Nakano, M., Ikeda, Y., Taniguchi, T., Yagi, T., Fuwa, M., Omi, N., Tokuda, Y., Tanaka, M., Yoshii, K., Kageyama, M., Naruse, S., Matsuda, A., Mori, K., Kinoshita, S., \& Tashiro, K.. (2009). Three Susceptible Loci Associated with Primary Open-Angle Glaucoma Identified by Genome-Wide Association Study in a Japanese Population. Proceedings of the National Academy of Sciences - PNAS, 106(31), 12838-12842. https://doi.org/10.1073/pnas.0906397106

Park, S., Jamshidi, Y., Vaideanu, D., BitnerGlindzicz, M., Fraser, S., \& Sowden, J. C. (2009). Genetic Risk for Primary Open-Angle Glaucoma Determined by LMX1B Haplotypes. Investigative Ophthalmology \& Visual Science, 50(4), 1522-1530. https://doi.org/10.1167/iovs.08-2483

Razeghinejad, M. R., \& Spaeth, G. L. (2011). A history of the surgical management of glaucoma. Optometry and vision science: official publication of the American Academy of Optometry, 88(1), E39-E47. https://doi.org/10.1097/OPX.0b013e3181fe22 26

Resende, A. F., Patel, N. S., Waisbourd, M., \& Katz, L. J. (2016). iStent ${ }^{\circledR}$ Trabecular Microbypass Stent: An Update. Journal of Ophthalmology, 2016, 2731856-2731859. https://doi.org/10.1155/2016/2731856

Souma, T., Tompson, S. W., Thomson, B. R. S., Owen M, K., Krishnakumar, Y., Shinji, F., Liang, L., Vachiranee, W., Kristina, N., Maurer-Stroh, S., Yanovitch, T. K., Luba, A., D. N., Finzi, S., Mauri, L., Javadiyan, S., Souzeau, E., Zhou, T., Hewitt, A. W., ... Young, T. L. (2016). Angiopoietin receptor TE mutations underlie primary congenital glaucoma with variable expressivity. The Journal of Clinical Investigation, 126(7), 2575-2587. https://doi.org/10.1172/JCI85830 
Stone, E. M., Fingert, J. H., Alward, W. L., Nguyen, T. D., Polansky, J. R., Sunden, S. L., Nishimura, D., Clark, A. F., Nystuen, A., Nichols, B. E., Mackey, D. A., Ritch, R., Kalenak, J. W., Craven, E. R., \& Sheffield, V. C. (1997). Identification of a gene that causes primary open angle glaucoma. Science (New York, N.Y.), 275(5300), 668-670. https://doi.org/10.1126/science.275.5300.668

Timberlake, G. T., Kennedy, M., (2005). "The Direct Ophthalmoscope How it Works and How to Use It." 2005. Retrieved from: https://web.media.mit.edu/ raskar/Eye/TheDir ectOphthalmoscope.pdf

Welsbie, D. S., Yang, Z., Ge, Y., Mitchell, K. L., Zhou, X., Martin, S. E., Berlinicke, C. A., Hackler, L., Jr, Fuller, J., Fu, J., Cao, L. H., Han, B., Auld, D., Xue, T., Hirai, S., Germain, L., Simard-Bisson, C., Blouin, R., Nguyen, J.
V., Davis, C. H., ... Zack, D. J. (2013).

Functional genomic screening identifies dual leucine zipper kinase as a key mediator of retinal ganglion cell death. Proceedings of the National Academy of Sciences of the United States of America, 110(10), 4045-4050. https://doi.org/10.1073/pnas.1211284110

Wiggs, J. L., \& Pasquale, L. R. (2017). Genetics of glaucoma. Human molecular genetics, 26(R1), R21-R27. ttps://doi.org/10.1093/hmg/ddx184

Zhou, C., Qian, S., Wu, P., \& Qiu, C. (2013). Anxiety and depression in Chinese patients with glaucoma: sociodemographic, clinical, and self-reported correlates. Journal of psychosomatic research, 75(1), 75-82. https://doi.org/10.1016/j.jpsychores.2013.03.0 05 
Appendix A: Figure 1. Anatomy of a healthy eye

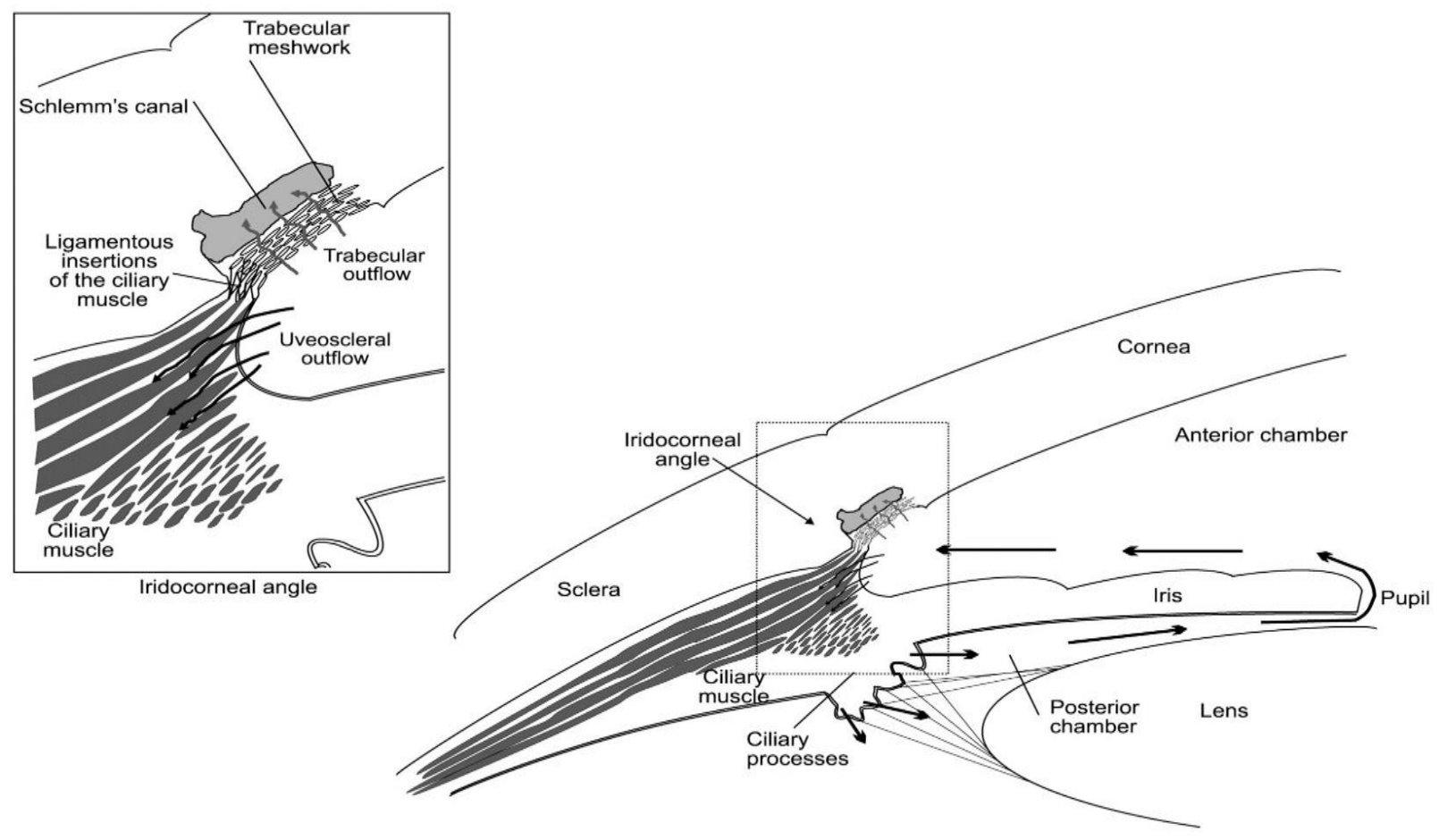

Note. In this figure, the normal cycle of aqueous humor throughout the eye is depicted. Starting from the ciliary processes and ending at Schlemm's canal (black arrows). From "Schematic Diagram of the Aqueous Humor Cycle” by Artur Llobet, Xavier Gaull, and Arcadi Gual licensed under CC BY 2.0. 
Appendix B: Figure 2. Development of Glaucoma

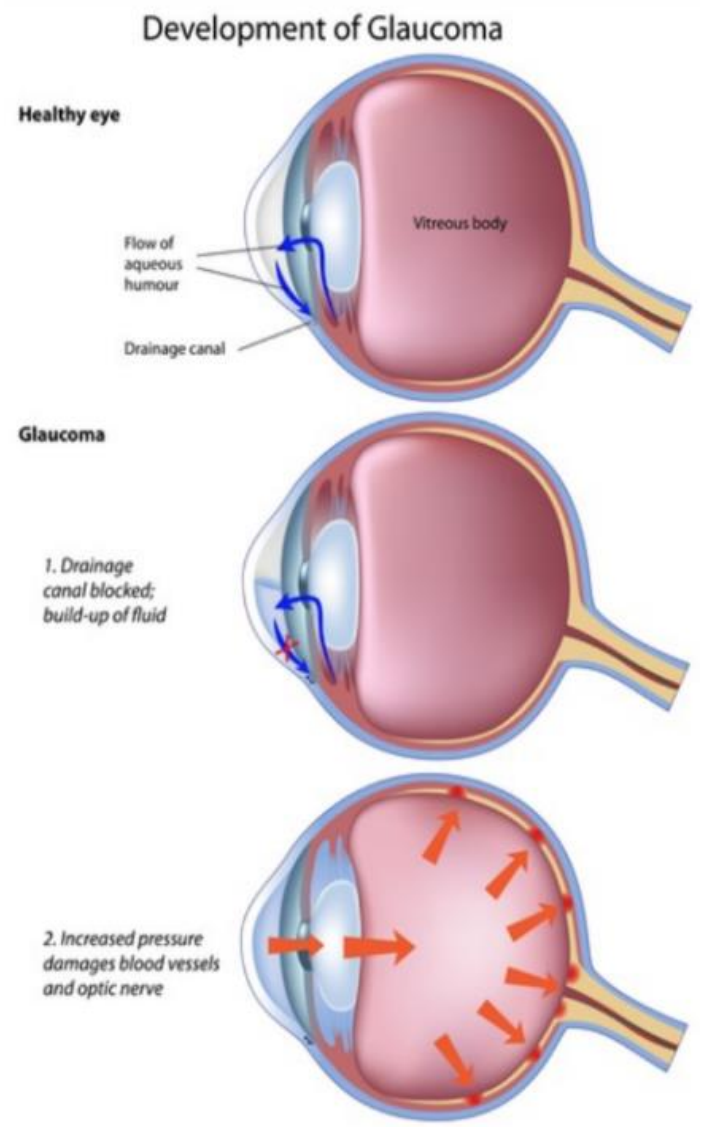

Note. This figure compares the flow of aqueous humor in a normal eye versus in an eye that develops Glaucoma due to blockage of the trabecular meshwork tissue and subsequent increase of IOP. From "Development of Glaucoma” by Alila Medical Media licensed under CC BY 2.0. 
Appendix C: Figure 3. POAG mechanism associated with the MYOC gene hotspot
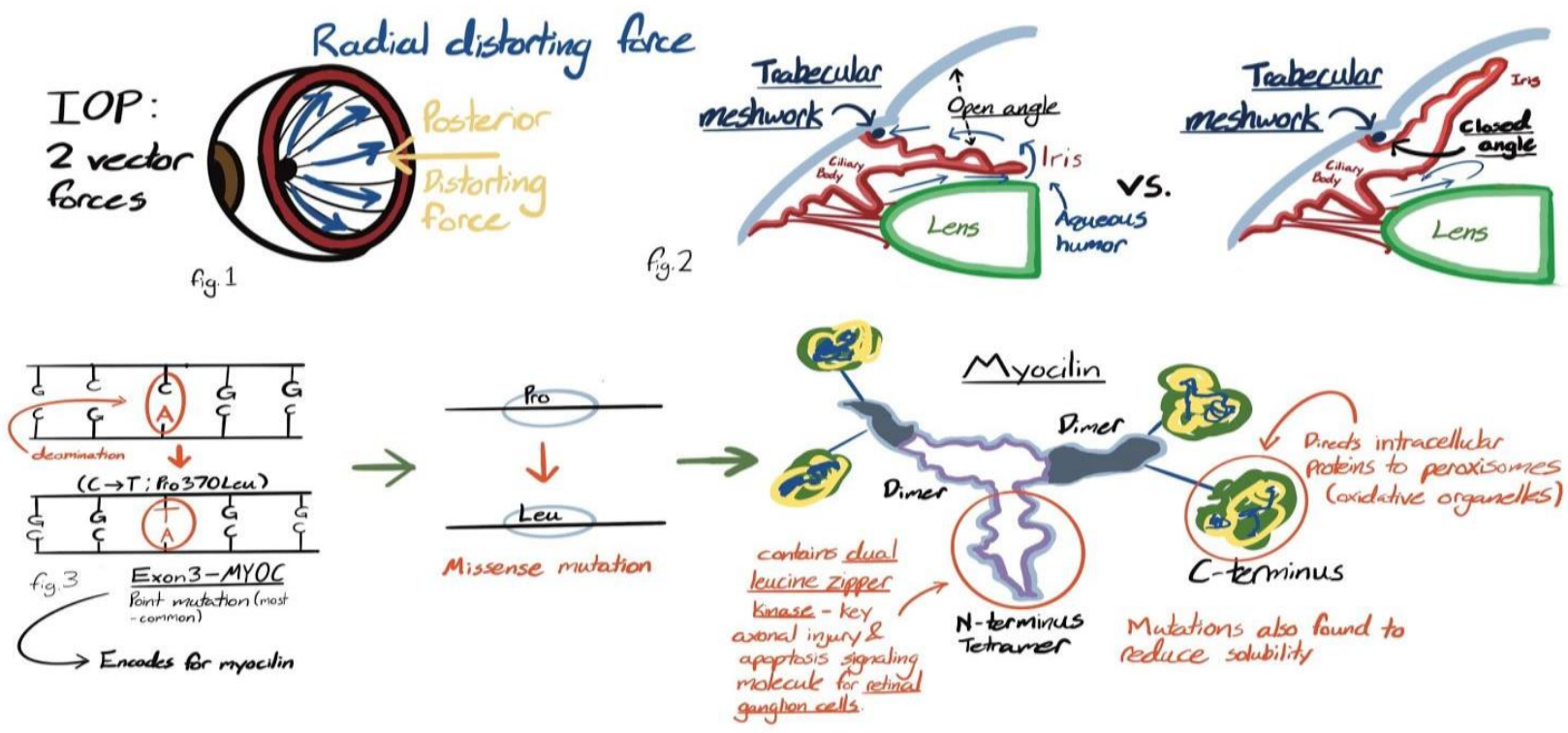

Note. Figure 3.1. Increasing IOP results in radial \& posterior distorting forces within the eye. Increased pressure induces apoptosis/damage retinal ganglion cells \& distort vision. Figure 3.2. The iris lies closer to the lens, exhibiting the characteristic, "open-angle" between the iris \& cornea (left). The presence of a "closed-angle", indicates the ciliary body \& iris' proximity to the cornea (right). In both diagrams, the trabecular meshwork is non-functioning or damaged in Glaucoma, preventing the efficient drainage of aqueous humor. Figure 3.3. Illustration of a point mutation on Exon 3-MYOC (gene implicated in POAG), resulting in a proline to a leucine missense mutation. The mutant Myocilin (protein) product may potentially exhibit defects in one or several key domains. 
Appendix D: Figure 4. Illustration of iStent function

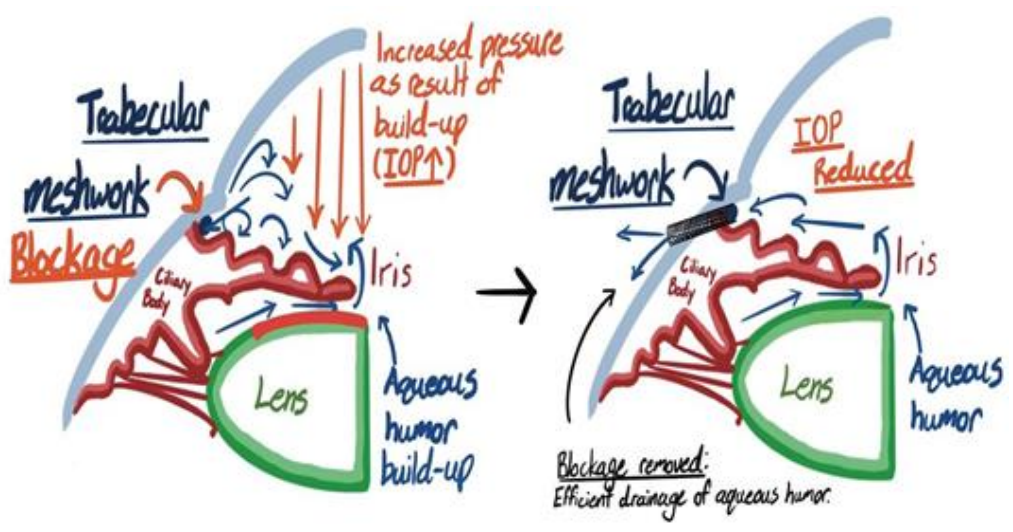

Note. Left panel: Dysfunctional trabecular meshwork causes a build-up of aqueous humor. Right panel: Installation of the iStent allows aqueous humor to properly drain; decreasing IOP. 
Appendix E: Figure 5. iStent anatomy

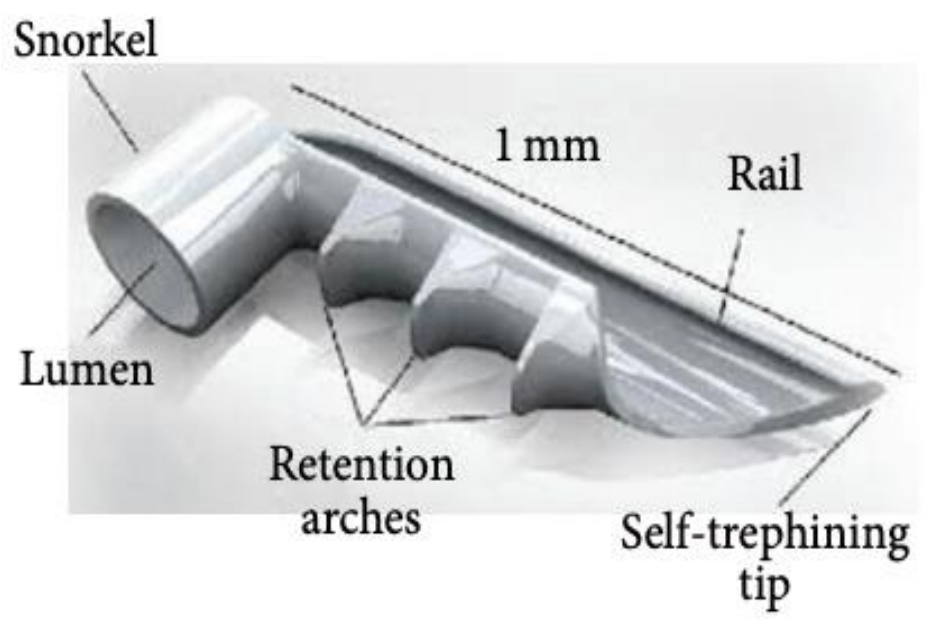

Note. The iStent anatomy allows for short \& permanent procedures. Beginning with insertion using a needlepoint self-trephining tip, retention arches secure the device in position, and allows aqueous humor to freely drain through the trabecular meshwork (right). From "iStent Anatomy" by The Eye Institute licensed under CC BY 2.0. 
Appendix F: Figure 6. iStent(s) in the trabecular meshwork
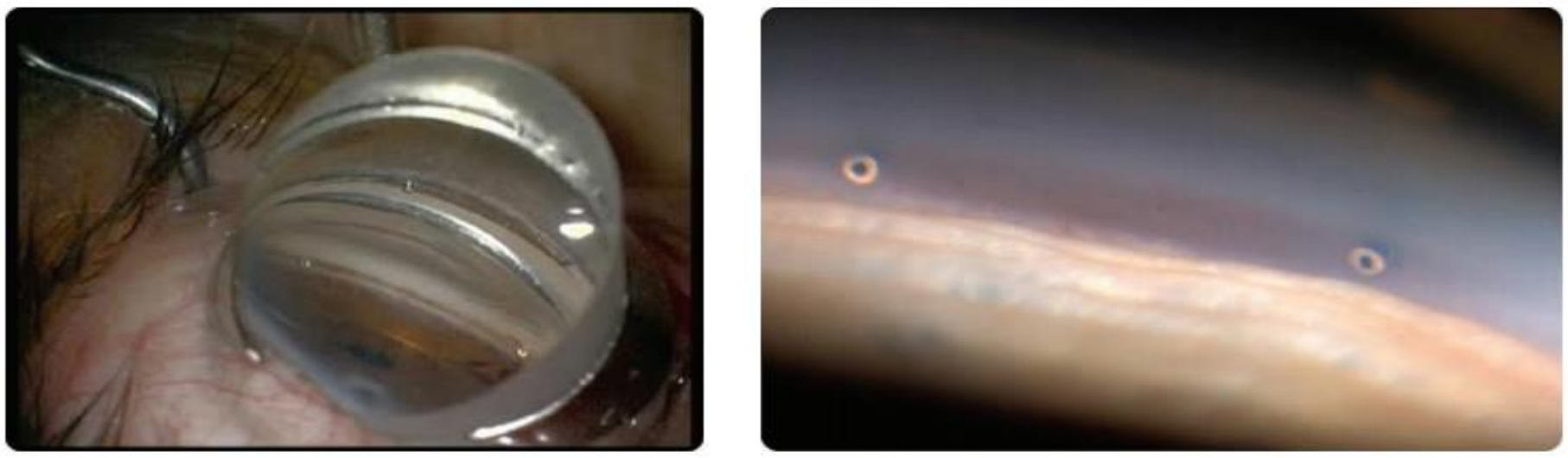

Note. Left panel: Image of direct ophthalmoscopy of the eye. Right panel: iStent(s) are installed within the trabecular meshwork which appears like white circles between the upper translucent layer (cornea) and lower beige layer (iris, ciliary body). From “Direct Gonioscopy and 2 Inserted iStents Under Gonioscopy” by Journal of Ophthalmology licensed under CC BY 2.0. 